\title{
Innate immunity in the central nervous system
}

\author{
Richard M. Ransohoff' and Melissa A. Brown² \\ ${ }^{1}$ Neuroinflammation Research Center, Lerner Research Institute, Cleveland Clinic, Cleveland, Ohio, USA. ${ }^{2}$ Department of Microbiology-Immunology, \\ Northwestern University Feinberg School of Medicine, Chicago, Illinois, USA.
}

\begin{abstract}
Immune responses in the CNS are common, despite its perception as a site of immune privilege. These responses can be mediated by resident microglia and astrocytes, which are innate immune cells without direct counterparts in the periphery. Furthermore, CNS immune reactions often take place in virtual isolation from the innate/adaptive immune interplay that characterizes peripheral immunity. However, microglia and astrocytes also engage in significant cross-talk with CNS-infiltrating $T$ cells and other components of the innate immune system. Here we review the cellular and molecular basis of innate immunity in the CNS and discuss what is known about how outcomes of these interactions can lead to resolution of infection, neurodegeneration, or neural repair depending on the context.
\end{abstract}

\section{Immune privilege: CNS innate immune cells do not phone home}

An essential function of innate immunity is to provide the informational input for adaptive immunity. In peripheral organs, innate DCs detect the presence and nature of pathogens (viral, bacterial, or protozoal; intracellular or extracellular) and, through the release of selective mediators, educate $T$ cells about the specifics of pathogen threat. Once the $\mathrm{T}$ cell has been informed (primed and polarized), it is directed to the site that harbors the pathogen (1-4). Here other resident or infiltrating innate cells decode the expressed array of $\mathrm{T}$ cell cytokines and, in a perfect immunological world, carry out the appropriate host attack on pathogen (Figure 1).

\section{Inflammation in the CNS: the role for DCs}

DCs play a critical role in initiating $T$ cell responses by taking up protein antigens in tissues, processing them into small peptides and then displaying them on their surface physically associated with MHC class II molecules. DCs migrate through afferent lymphatics to draining lymph nodes and present antigen to naive or memory T cells. Importantly, there is no evidence that DCs with such capacities reside in the healthy CNS parenchyma, nor do CNS resident immune cells prime naive T cells (reviewed in ref. 5). Cells carrying DC surface markers (e.g., CD11b, CD11c) are readily detected in the meningeal coverings of the CNS and in the choroid plexus, the site of cerebrospinal fluid synthesis (6). Although cells with DC markers are abundant in the inflamed CNS parenchyma, they are primarily observed after blood-brain barrier (BBB) disruption, suggesting that many are peripherally derived while others represent resident microglia induced to express such markers by locally expressed cytokines (7-10).

The lack of parenchymal DCs and the fact that no other parenchymal CNS cells fit the operational definition of a DC (antigen uptake, migration to draining lymph nodes, and presentation to naive T cells) constitute the cellular basis of CNS immune privilege. Immune privilege of the CNS, a hoary concept whose definition has become swollen and imprecise over more than six decades can be reduced to two observations: (a) immunogens such as xenografts, viruses, or bacterial lysates fail to elicit adaptive immune responses following non-traumatic micro-injection into the CNS parenchyma and (b) peripheral immunization with the same immunogen leads to a brisk immune response to the CNS depot of antigen.

Conflict of interest: The authors have declared that no conflict of interest exists. Citation for this article: J Clin Invest. 2012;122(4):1164-1171. doi:10.1172/JCI58644.
Why is CNS tissue immune privileged? Two possibilities are salient: (a) robust intrathecal inflammatory reactions can damage delicate, non-regenerating post-mitotic cells such as neurons and oligodendrocytes, suggesting that the lack of adaptive immune responses might confer a survival advantage; and (b) pathogen ingress into the CNS always involves transit from a peripheral site of entry that will first elicit a response in the draining lymph nodes or spleen. Therefore, it would be redundant to endow the CNS with the ability to generate adaptive immune responses de novo.

The BBB has its phylogenetic origin in invertebrates and evolved to provide a precisely calibrated chemical and ionic environment to optimize neuronal function. Yet the BBB is also well suited to restrain CNS inflammation by excluding plasma proteins as well as peripherally derived innate and adaptive immune cells and their associated inflammatory molecules $(11,12)$. Additionally, the parenchymal CNS environment is anti-inflammatory, featuring high local concentrations of inflammation-suppressive cytokines such as TGF- $\beta$ and IL-10 and is replete with gangliosides, which can be toxic to T cells (13-17).

Cumulatively, the lack of resident DCs and the relative antiinflammatory environment of neural tissue lead to innate immune processes that are muted and secluded within the CNS. There is no efficient outward migration of CNS innate immune cells to sound the alarm in lymphoid organs, requiring that resident innate immune cells deal directly with pathogens and tissue damage. Under many circumstances resident cells recruit inflammatory cells from the circulation and interact with these cells to facilitate vigorous inflammatory responses.

Recognizing and responding to microbial pathogens is the cardinal function of innate immune cells. Basic host defense mechanisms are operational in microglia and astrocytes, despite their sequestration within the CNS. Host defense begins with recognition of structural signatures characteristic of pathogens (reviewed in refs. 18-20). Microbial warnings are mediated by pathogenassociated molecular patterns (PAMPs) and include bacterial, viral, and protozoal products (protein, lipid, nucleic acid, carbohydrate). PAMPs are recognized by TLRs, which reside on the plasma membrane or in endosomal compartments (21). In a prototypical scenario, the engagement of TLRs evokes NF- $\mathrm{kB}$ activation, resulting in increased transcription of genes encoding IL-1 family cytokines (Figure 2). Pro-forms of resulting peptides, for example proIL-1 $\beta$, remain cytoplasmic until cleaved enzymatically by activated caspase-1, releasing active IL-1 $\beta$ (22). 
A

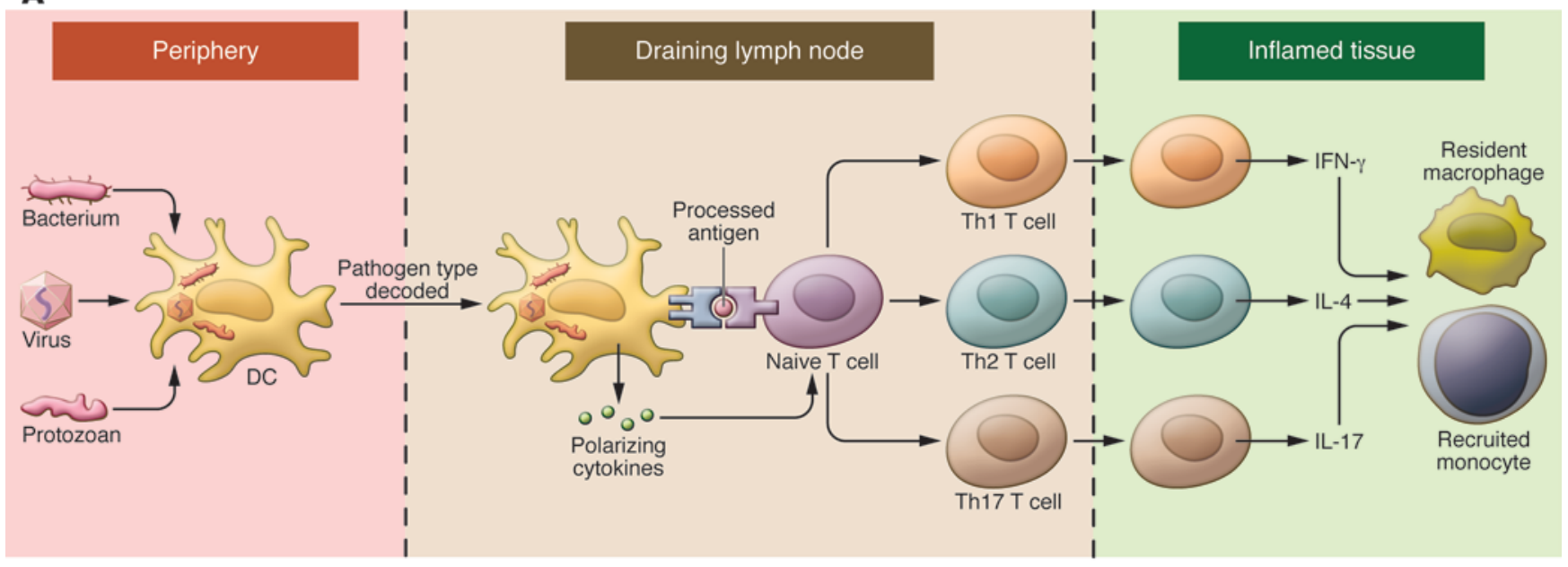

B

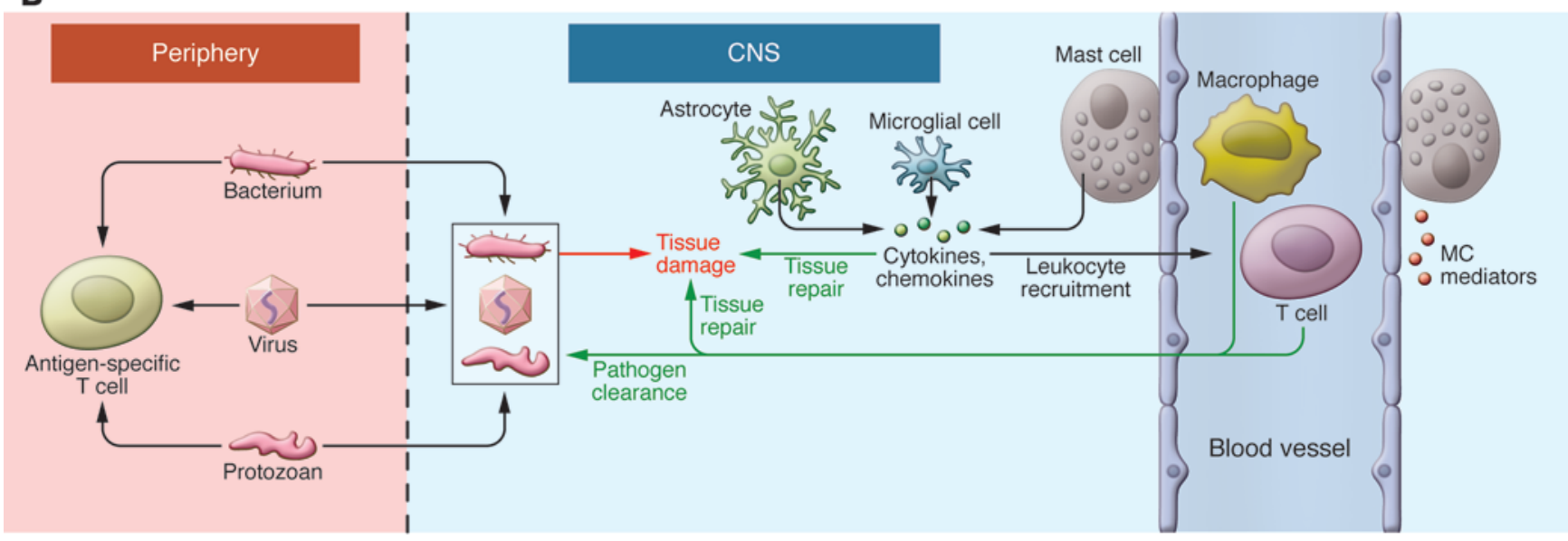

Figure 1

Innate immunity in the periphery and CNS. (A) In the face of a peripheral infection, innate immune cells prime and instruct T lymphocytes. Tissue DCs internalize microbial protein antigens, process them into peptides, and display them on their surfaces with MHC class II molecules; migrate to draining lymph nodes; and present antigens to naive CD4+ T cells. DCs direct the quality of the subsequent inflammatory response by decoding distinctive pathogen-associated signals and transmitting this information to T cells in the form of regulatory cytokines such as IL-12 (for Th1), IL-4 (Th2), or IL-6/TGF- $\beta$ (Th17). In addition, Iymph node environmental cues can provide information about the site of infection (gut, skin, or other). Armed with this information, effector T cells migrate to infected tissues. Upon reactivation, Th1, Th2, and Th17 cells express phenotype-defining cytokines that act on resident and recruited innate cells, which operate collectively with factors such as complement to clear the infection. (B) Resident microglia and astrocytes exert multiple functions in the CNS, including protective and restorative responses to CNS infection or injury. Cytokines and chemokines expressed by resident CNS cells also promote the recruitment of circulating lymphocytes and myeloid cells from the periphery to assist in pathogen clearance. Innate responses in the CNS cannot directly initiate adaptive immunity. Innate CNS reactions also occur during neuroinflammatory disorders and utilize many of the same components as do host defense responses.

Activation of caspase- 1 is initiated by signaling from a second set of innate immune receptors, termed "nucleotide-binding domain leucine-rich repeat-containing (LRR-containing) receptors" (NLRs), whose function is dependent on the assembly of large ( 700-kDa) complexes termed "inflammasomes" $(23,24)$. NLRs have been studied extensively in hematopoietic cells including myeloid lineage cells in the CNS such as microglia. Our understanding of specific NLR functions is encumbered by an unwieldy and ever-changing terminology. The largest NLR subfamily (with 14 members), and the one most pertinent for neuroinflammation, is designated the NACHT domain-, LRR domain-, and pyrin domain-containing protein (NALP) family (25). Inflammasomes, defined by their core NALPs, are activated by the cytoplasmic presence of specific microbial components, tissue-injury products, or inflammation-associated metabolic alterations including low cytosolic potassium (26). For NALP3 inflammasomes, effective stimuli include bacterial muramyl dipeptide, bacterial RNA, ATP, and uric acid. Inflammasomes recruit and activate caspase-1, thereby complementing TLR signaling to generate mature IL-1 $\beta$ and IL-18. Another family member, IL-33, is sequestered within cell nuclei, released by cell injury, and inactivated by caspase- 1 cleavage (27-29). Along with IL-1 $\alpha$, IL-33 is considered an alarmin (indicator of cell damage) (30).

Dissection of TLR and NLR signals involved the convergence of two distinct lines of research. Toll and spätzle, the index TLR family receptor/ligand pair, were discovered as regulators of Dro- 


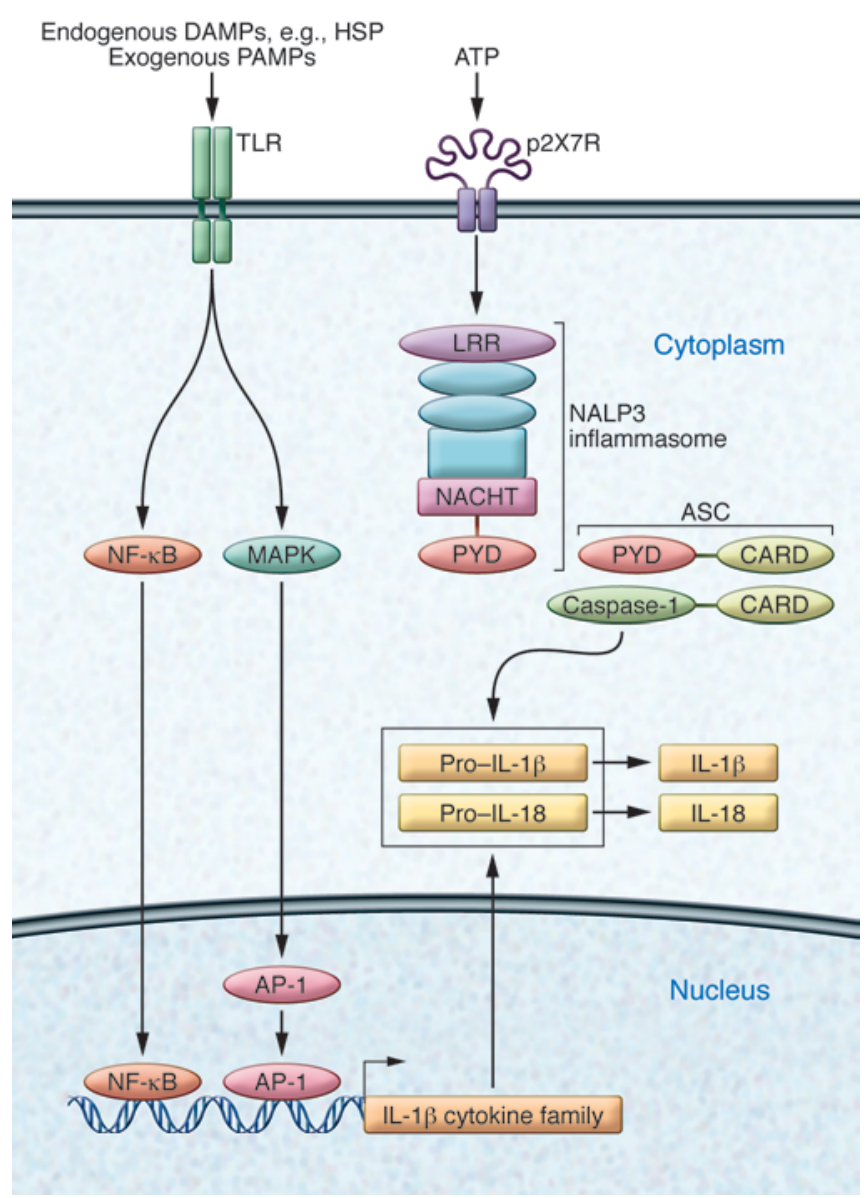

sophila dorsal-ventral patterning and, later, antifungal immunity $(18,21,31)$. NALP3, also known as cryopyrin, was characterized as the mutated gene in autosomal-dominant autoinflammatory disorders such as Muckle-Wells syndrome and familial cold autoinflammatory syndrome, typified by excess IL-1 $\beta$ activity and effectively treated by IL- $1 \beta$ sequestration $(22,25)$. These pathways were linked by the discovery that cooperative signaling through TLRs and NLRs culminated in secretion of IL-1 family cytokines (32).

The interplay of TLR and NLR signaling effectively protects against pathogens, and both receptor families are expressed in resident CNS cells that participate in innate immunity. Microglia, myeloid cells of the CNS, express all TLRs (33). A more restricted array is expressed in astrocytes $(34,35)$. Upon pathogen exposure, activated microglia secrete biologically active IL- $1 \beta$ and IL-18 through expression of NLR-mediated inflammasome activity, which in turn elicits production of a secondary inflammatory cytokine cascade by both microglia and astrocytes $(23,36,37)$. For example, IL-1 $\beta$ can induce expression of TNF- $\alpha$ and IL- 6 , while IL-18 stimulates production of IL-17. Inflammatory cytokines also diminish BBB barrier function and enhance recruitment of hematogenous leukocytes (38).

\section{Innate recognition of tissue injury: variation on a theme}

TLRs and NLRs are also highly effective at sensing and responding to non-infectious sterile tissue injury, as observed in stroke or trauma (Figure 2). Just as pathogens are detected by virtue of releasing "stranger" signals, so do damaged cells release "dan-

\section{Figure 2}

Innate recognition of infection or tissue injury. Endogenous DAMPs such as HSP or exogenous bacterial PAMPs activate the innate immune cells of the CNS by engaging cell surface receptors such as TLRs. Ligation of TLRs initiates an intracellular signaling cascade that involves activation of NF-KB and MAPK activity, which leads to formation of transcription factor AP1. Together these components drive transcription of IL-1 family cytokine precursor proteins pro-IL-1 $\beta$ and pro-IL-18. NLRs are activated by cytosolic microbial products, changes in cytosolic $\mathrm{pH}$, or potassium levels often associated with stimulation of the ligand-gated ion channel P2X7R by extracellular ATP. NLRs such as NALP3 are central constituents of inflammasomes, multiprotein complexes that mediate activation of caspase-1, which catalyzes cleavage and maturation of IL-1 family cytokines. Functional domains of inflammasome components include caspase activation and recruitment domains (CARDs), which are present in both caspase- 1 and the adapter apoptosis-associated Speck-like protein containing CARD (ASC). PYD, pyrin domain.

ger" signals, designated damage-associated molecular patterns (DAMPs). TLRs and NLRs sense DAMPs: TLR3, TLR7, and TLR9 detect microbial nucleic acids and also those released from necrotic cells (39). TLR2 and TLR4 respond to cellular hsp such as Hsp60, Hsp70, and $\alpha \mathrm{B}$ crystallin. NLRs can be activated by endogenous cellular products such as uric acid crystals (as in gouty arthritis) and aggregated peptides $(20,40)$. ATP from damaged cells activates purinergic receptor-regulated channels to cause cytosolic ion fluxes that are detected by NLRs $(41,42)$.

\section{Cellular soldiers of CNS innate immunity}

Microglia. Microglia, the archetypal cells of CNS innate immunity (43), are a unique myeloid cell population, derived from the yolk sac during a narrow time window before vascularization or definitive hematopoiesis in the embryo (44). Once established in the CNS parenchyma, microglia are sustained by proliferation of resident progenitors, independent of blood cells (45). In vitro, microglial activation by diverse stimuli (46) induces varied programs of gene expression, yet these gene-expression patterns have not been validated in vivo (47). Activation of microglia is accompanied by morphological changes (Figure 3 and ref. 48). Despite their dissimilar embryonic origins, microglia are related to resident tissue macrophages. Monocyte-derived macrophages are classified as M1, M2a, M2b, and M2c subsets $(49,50)$. It is plausible that microglia also transcribe context-dependent, activation-related genes that confer unique phenotypes, however the M1/M2 paradigm has not been extended to any tissue-resident macrophages, let alone a population as unusual as microglia. Repurposing techniques including parabiosis (51) might help in accurately defining subsets of microglia (reviewed in ref. 52).

Systemic inflammation also activates microglia (53-57). Paradoxically, microglial responses to innate stimuli such as systemic LPS show interesting neuroprotective properties in experimental systems. In this paradigm, (stress preconditioning), systemic challenges elicit cytokine responses, which activate microglia and ameliorate injury after subsequent CNS insults including stroke or physical trauma (58-61). The molecular bases and clinical relevance of stress preconditioning remain uncertain.

Chronic neurodegeneration also leads to microglial activation, although the outcome of the activation may be beneficial, deleterious, or neutral. Neurons constitutively express cell-surface and secreted microglial inhibitors; it is conceivable that neuronal cell 
A

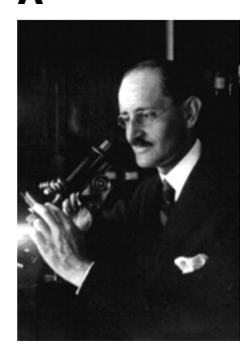

B

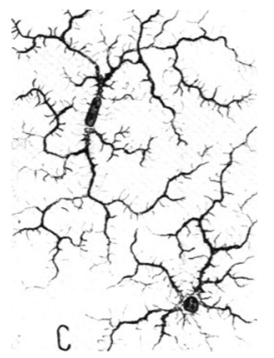

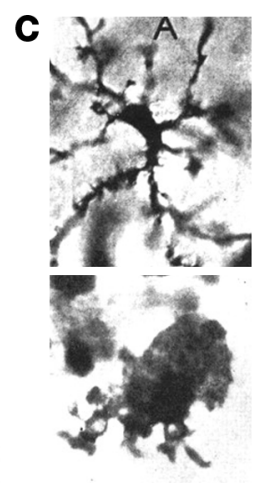

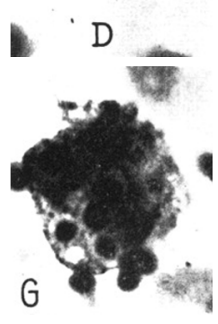

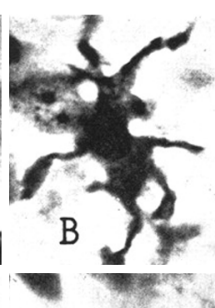
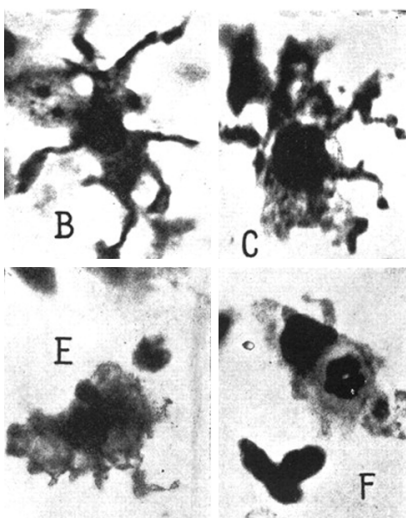

$F$

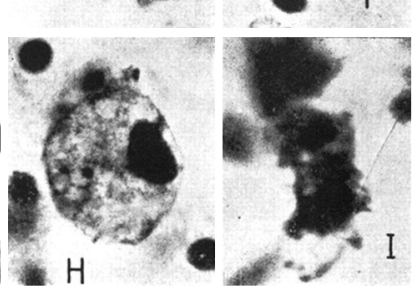

\section{Figure 3}

Microglial cells, as described by Pio del Rio-Hortega (161). (A) Pio del Rio-Hortega (1882-1945), who characterized and named microglial cells. (B) Images of ramified microglial cells drawn by Hortega. (C) Morphological transformation of microglia to phagocytic macrophage. Panels as lettered in C: A, Microglial cell with modestly thickened processes as compared with ramified microglia; $\mathrm{B}$, microglial cell with short, thick processes and enlarged cell body; C, microglial cell with pseudopodia; $\mathrm{D}$, amoeboid microglial cell; E, amoeboid microglial cell with pseudopodia; F, microglial cell with phagocytosed leukocyte; $\mathrm{G}$, microglial cell with numerous phagocytosed erythrocytes; $\mathrm{H}$, microglial cell with lipid inclusions, also termed "foam cell"; I, microglial cell in mitotic division. Reproduced with permission from Physiological Reviews (161). death or injury removes this suppression (46). If so, the microglial response to neurodegeneration represents a specialized danger signal. Genetic models have unraveled certain microglial contributions to neurodegeneration. In a genetic mouse model of motor neuron disease, targeted deletion of the causative mutant superoxide dismutase gene in microglia remarkably prolonged the lifespan of the mice even though the mutant transgene was still expressed by neurons and astrocytes (62). Targeted ablation of the CX3CR1 chemokine receptor gene (expressed in the CNS only by microglia) modulates microglial reactivity, in most cases increasing cytokine production and effector functions (63). CX3CR1-deficient mice show enhanced amyloid clearance in Alzheimer's disease (AD) amyloid deposition models (64), consistent with beneficial activation of microglia $(52,63,65,66)$. By contrast, CX3CR1 deficiency worsens toxicity in $\tau$ hyperphosphorylation models (67) and in models incorporating both pathologies, and complex effects are seen in models incorporating both pathologies (68). Amyloid $\beta$, the principal component of AD-associated amyloid, directly activates microglia through the NALP3 inflammasome pathway (36), and subsequent production of IL-1 $\beta$ may worsen neurodegeneration (69).

Astrocytes. With the exception of microglia and mast cells, CNS resident cells descend from neuroepithelial stem cells and are categorized as neurons and glia, with glia further subdivided into astrocytes, oligodendrocytes, and polydendrocytes. A traditional view holds that glia exist to serve and protect neurons. However, neurons and glia function in intimate interconnections to support every aspect of brain development and function (as reviewed in refs. 70-74). Astrocytes are the best-characterized innate immune neuroglia. The main functions of astrocytes include buffering CNS potassium, removing and recycling potentially toxic glutamate, adjusting water balance, and modulating synaptic activity and blood flow. Astrocytes also produce neurotrophins and antiinflammatory cytokines such as IL-10 (75).

Upon activation by TLR and NLR signals, astrocytes participate in innate immune reactions and are the principal CNS sources of innate inflammatory mediators, including several complement components, IL-1 $\beta$, IL-6, and chemokines such as CCL2, CXCL1, CXCL10, and
CXCL12 (76-89). Essential homeostatic functions of astrocytes are compromised during inflammatory reactions, potentially worsening outcomes. For example, CXCL12 signaling to astrocytes promotes physiological release of glutamate during synaptic transmission, and also induces release of small amounts of TNF- $\alpha$. In inflammatory conditions, CXCL12 plus TNF- $\alpha$ signal to microglia to produce large quantities of TNF- $\alpha$. This cytokine, at high concentrations, impairs the capacity of astrocytes to detoxify glutamate, resulting in neuronal loss through a mechanism termed "excitotoxicity" (79, 90, 91). Microglial-astrocyte interactions are also critical in CNS innate immunity. The deciphering of microglial-astrocyte communication at the molecular level is still in its infancy but already shows promise for identifying interesting therapeutic targets $(92,93)$.

In a mouse model, the inflammatory transcriptional regulator $\mathrm{NF}-\kappa \mathrm{B}$ was silenced in astrocytes by transgenic overexpression of a

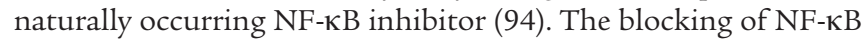
signaling in astrocytes showed benefit in disease and injury models - reduced retinal ganglion cell death after ischemic injury; improved recovery from spinal cord trauma, along with increased axonal sparing and regeneration; and lessened inflammation in EAE, a rodent model of the human inflammatory demyelinating disease MS. These findings highlighted the contributions of astrocyte-specific inflammatory signaling for a multitude of CNS pathologies (94-98).

\section{Interactions between innate immune cells and $\mathrm{T}$ cells in the CNS}

CNS innate immune cells respond to primed $T$ cells and their cytokine directives. Under $\mathrm{T}$ cell-mediated inflammatory conditions, the CNS admits large numbers of peripheral innate immune cells. Indeed, CNS infiltration by peripheral cells is critical for protective host defense against infection and for repair after stroke or physical trauma (99-104). However, restraint is required because hematogenous inflammation causes profound damage if the reaction is excessive or inappropriate. The interaction of the CNS innate immune system with infiltrating T cells is typified by MS and EAE (reviewed in refs. 105, 106). EAE can be induced by actively immunizing rodents with myelin protein peptides, which are emulsified 
in immune-stimulating adjuvants. IFN- $\gamma$-producing Th1 cells and IL-17-producing Th17 cells subsequently accumulate in the CNS and initiate demyelination. This immunization protocol also generates $T$ cells that cause disease upon adoptive transfer to naive recipients, a process termed "passive immunization" (107). Myelin-specific $\mathrm{CD}^{+} \mathrm{T}$ cells are found in peripheral blood of healthy individuals and in MS patients $(108,109)$. Clonally expanded and potentially autoreactive $\mathrm{CD}^{+}$and $\mathrm{CD} 8^{+} \mathrm{T}$ cells have been detected at autopsy in CNS tissues from individuals with MS but not in relevant controls $(110,111)$. Thus it is likely that, in MS as in EAE, disease-causing $\mathrm{T}$ cells are initially activated in peripheral lymphoid organs, where they undergo differentiation and expansion. When autoreactive $\mathrm{T}$ cells are reactivated in the CNS by cognate antigen, release of immune mediators facilitates an extensive local inflammatory reaction, which abrogates the trafficking restraints and barrier functions of the BBB. Large-scale CNS infiltration by inflammatory cells and entry of plasma proteins culminates in demyelination, edema, compromise of neural cell function, and neurobehavioral impairment.

\section{How do T cells communicate with resident and infiltrating innate cells?}

$\mathrm{T}$ cell adoptive transfer experiments demonstrate that encephalitogenicity of Th cells is exquisitely dependent on their production of GM-CSF - even though GM-CSF-deficient Th cells enter CNS and produce other cytokines, disease does not develop. GM-CSF is required to recruit $\mathrm{CD} 11 \mathrm{~b}^{+}$myeloid cells, which are thought to sustain local CNS inflammation, mediate direct myelin damage, and support the survival of lymphocytes (112-114).

Microglia express IL-23 and IL-1 $\beta$, both of which promote GMCSF expression by $\mathrm{T}$ cells (115), implicating microglial- $\mathrm{T}$ cell interactions in intrathecal $\mathrm{T}$ cell survival and effector function. In transgenic mice, EAE was less severe if microglial responses were impaired (116). Antibodies to GM-CSF are being utilized in clinical trials of inflammatory diseases so that translational applications of this line of research are feasible (117).

\section{Neutrophils}

That neutrophils are considered the first line of defense against extracellular and intracellular bacteria is illustrated by life-threatening conditions that result from neutrophil deficiency $(118,119)$. Neutrophils are rapidly mobilized from the bone marrow in response to signals from CXC family chemokines to mediate pleiotropic functions in immune-inflammatory responses (reviewed in ref. 120). Neutrophils respond to PAMPs and DAMPs through TLRs and NLRs and are also activated by cytokines such as TNF- $\alpha$ and IFN- $\gamma$. Once activated, neutrophils upregulate CD15 and CD11b, adhesion molecules that enhance their association with endothelium and migration into tissues (120). Activated neutrophils also produce reactive intermediates through their vigorous respiratory burst and release a plethora of pre-formed mediators: cytokines, chemokines, colonystimulating and angiogenic factors, lytic enzymes, and antimicrobial peptides. Neutrophils influence lymphocyte migration as well; TNF- $\alpha$-induced production of CXCL9 and CXCL10 or CCL20 by neutrophils recruits Th1 or Th17 cells, respectively (121-123). Neutrophil-lymphocyte interactions induce survival factors that prolong the lifespan of the short-lived neutrophils. Adding to the inflammatory cascade, T cells recruit neutrophils by secreting IL-17 (124).

Neutrophils are implicated in inflammatory conditions of the CNS. Bacterial meningitis elicits neutrophil infiltration, which is often associated with unfavorable outcomes, potentially because of the severity of the infection (125). Roles of neutrophils in chronic sterile neuroinflammation (as in MS) are under investigation. G-CSF, a growth factor that supports neutrophil activation, worsens MS disease activity (126). Neutrophils are not detected in postmortem MS tissues, nor are there increased neutrophils in the blood or CSF of MS patients (127). By contrast, lesions of neuromyelitis optica (NMO), an autoimmune CNS disease caused by aquaporin 4 antibodies, show abundant neutrophils, which may also be found in CSF during active disease (128). Variable acuity of $\mathrm{NMO}$ and MS may contribute to these different findings. NMO lesions are much more destructive and more likely to cause death during acute disease, whereas fatal outcomes of MS occur through complications of immobility after decades of disease. Therefore, the absence of neutrophils in lesions of MS (studied at autopsy) may not be proof of their absence during lesion formation.

Animal models also implicate neutrophil involvement in MS. In EAE, neutrophils are among the earliest CNS-infiltrating cells $(129,130)$, and neutrophil depletion reduces EAE severity dramatically (131). Furthermore, CXCR2 $2^{-/}$mice are resistant to EAE induction $(131,132)$.

Neutrophil influx into the CNS during EAE results from TNF- $\alpha$ production by meningeal mast cells (133). Because neutrophils also promote B cell survival and proliferation (120), innate neutrophils and mast cells might contribute to the B cell follicle-like structures that are found at autopsy in the meninges of MS tissues $(134,135)$.

\section{Mast cells}

Mast cells are myeloid cells defined by c-kit ${ }^{\text {hi }} \mathrm{FcERI}^{+}$expression and are well known for roles in allergic disease and host defense (136, 137). Mast cells are particularly numerous within tissues exposed to the external environment, such as skin, gut, and respiratory tract, but are also found in brain, spinal cord, and meninges. Classic antimicrobial mast cell responses involve the release of TNF- $\alpha$ and IL-1 $\beta(136,138-140)$.

Collectively, mast cells comprise a large population of CNS cells, yet they are fixed and widely dispersed, which poses hurdles for direct study. Nevertheless, provocative correlative findings have been reported that implicate these cells in CNS inflammation. Mast cells are present in active MS plaques $(141,142)$, and mast cell-specific transcripts encoding tryptase and FceRI are detected in lesions of chronic MS (143). Tryptase and histamine are present in the CSF of MS patients but not healthy individuals $(144,145)$. Mast cells in the CNS parenchyma likely contribute to local inflammatory responses, and CNS mast cells appear to exert both neuroprotective and damaging effects following concussion injury or stroke (146).

There are limitations to the commonly used experimental models that utilize c-kit-mutant, mast cell-deficient mice (147) for the study of mast cell function, as mast cell development is exquisitely dependent on SCF signaling through c-kit. Mice with reduced SCF signaling due to mutations in the c-kit receptor $(\mathrm{W} / \mathrm{Wv}$ or Wsh mice) exhibit a loss of mast cells. Mast cells can be reconstituted by systemic or local transfer of bone marrow-derived mast cell precursors in mice harboring c-kit mutations. The c-kit-mutant mice have additional hematologic and developmental abnormalities, and it is therefore essential to use mast cell reconstitution to confirm that the observed phenotypic differences between wild type and Kit mutant mice are mast cell dependent $(148,149)$. Unfortunately, transferred mast cells fail to reconstitute the brains and spinal cords of c-kit mutant mice, making it challenging to use this model to address the functions of CNS-resident mast cells in health or disease (147). 
Initial EAE studies using $\mathrm{c}-\mathrm{kit}^{\mathrm{W} / \mathrm{W}_{\mathrm{v}}}$ or c-kit ${ }^{\mathrm{W} s \mathrm{sh}}$ mice employed diverse disease-induction protocols and subjective neurobehavioral scoring, yielding inconsistent and conflicting conclusions about EAE severity in mast cell-deficient mice (150-153).

Objective, quantitative disease severity measures revealed that mast cells were critical for fulminant disease in both chronic (C57BL/6-MOG $35-55$-induced) and relapsing remitting (SJL$\mathrm{PLP}_{139-151}$-induced) EAE $(133,154)$. BBB integrity was enhanced and decreased inflammatory cell infiltrates were decreased in $c^{-k i t}{ }^{\mathbb{W} / \mathbb{W v}_{v}}$ mice with EAE, as compared with wild-type animals (133). Interestingly, TNF- $\alpha$ production by dura mater and pia mater mast cells regulates BBB function as well as $\mathrm{T}$ cell and myeloid cell infiltration into the CNS (133), consistent with the idea that these mast cells are protective first responders to microbial CNS challenge, as they are in the periphery.

During the preclinical phase of EAE, T cells interact with APCs and proliferate in the leptomeninges around the spinal cord, suggesting that the leptomeninges is a site of $\mathrm{T}$ cell reactivation (155-158). Given that mast cells direct immune cell traffic to peripheral sites of infection (159), it is tempting to speculate that meningeal mast cells may also promote cellular cross-talk between $\mathrm{T}$ cells and myeloid cells in EAE. Determination of how CNS parenchymal and meningeal mast cells contribute both to protective CNS immunity and to harmful neuroinflammation now appears critical for identifying effective and comprehensive therapies for CNS diseases. The ongoing development of novel genetic murine models with selective mast cell defects will help toward this goal (160).

1. Edele F, et al. Cutting edge: instructive role of peripheral tissue cells in the imprinting of $\mathrm{T}$ cell homing receptor patterns. J Immunol. 2008; 181(6):3745-3749.

2. Dudda JC, et al. Dendritic cells govern induction and reprogramming of polarized tissue-selective homing receptor patterns of $\mathrm{T}$ cells: important roles for soluble factors and tissue microenvironments. Eur J Immunol. 2005;35(4):1056-1065.

3. Mora JR, Cheng G, Picarella D, Briskin M, Buchanan N, von Andrian UH. Reciprocal and dynamic control of CD8 $\mathrm{T}$ cell homing by dendritic cells from skin- and gut-associated lymphoid tissues. J Exp Med. 2005;201(2):303-316.

4. Oyoshi MK, et al. Epicutaneous challenge of orally immunized mice redirects antigen-specific gut-homing $\mathrm{T}$ cells to the skin. J Clin Invest. 2011; 121(6):2210-2220

5. Galea I, Bechmann I, Perry VH. What is immune privilege (not)? Trends Immunol. 2007;28(1):12-18.

6. Prodinger $\mathrm{C}$, et al. CD11c-expressing cells reside in the juxtavascular parenchyma and extend processes into the glia limitans of the mouse nervous system. Acta Neuropathol. 2011;121(4):445-458.

7. Miller SD, McMahon EJ, Schreiner B, Bailey SL. Antigen presentation in the CNS by myeloid dendritic cells drives progression of relapsing experimental autoimmune encephalomyelitis. Ann N Y Acad Sci. 2007;1103:179-191.

8. Ercolini AM, Miller SD. Mechanisms of immunopathology in murine models of central nervous system demyelinating disease. J Immunol. 2006; 176(6):3293-3298.

9. McMahon EJ, Bailey SL, Miller SD. CNS dendritic cells: critical participants in CNS inflammation? Neurochem Int. 2006;49(2):195-203.

10. Almolda B, Gonzalez B, Castellano B. Antigen presentation in EAE: role of microglia, macrophages and dendritic cells. Front Biosci. 2011;16:1157-1171.

11. DeSalvo MK, Mayer N, Mayer F, Bainton RJ. Physiologic and anatomic characterization of the brain surface glia barrier of Drosophila. Glia. 2011;

\section{Summary}

The immune-privileged status of the CNS has evolved to maintain homeostasis required for neural function and host defense. The inability to generate robust and potentially harmful adaptive immune responses therefore requires a primary reliance for host defense on the sequestered and moderate innate responses of microglia, astrocytes, and other resident innate cells. Nonetheless, pathologic neuroinflammation is inherent in all diseases, which disrupt CNS tissue elements, including MS, AD, Parkinson's disease, stroke, and traumatic brain injury. Our understanding of the interactions between resident and peripheral immune cells, neurons, and glial cells and their implications for host defense, tissue repair, and neurodegeneration is still in its infancy. However, the delineation of the molecular interactions between the immune and nervous systems is proceeding rapidly and will yield translational application in the years to come.

\section{Acknowledgments}

Research in the Ransohoff lab is supported by the NIH (grants R0NS132151, R21NS78420, and K24NS51400), the National MS Society (RG4550), gifts from the Walker and Warren families, and the Williams Family Foundation for MS Research.

Address correspondence to: Richard M. Ransohoff, Neuroinflammation Research Center, Lerner Research Institute, Cleveland Clinic, Mail Code NC30, 9500 Euclid Avenue, Cleveland, Ohio 44195, USA. Phone: 216.444.0627; Fax: 216.444.7927; E-mail: ransohr@ccf.org.
59(9):1322-1340

12. Banerjee S, Bhat MA. Neuron-glial interactions in blood-brain barrier formation. Annu Rev Neurosci. 2007;30:235-258

13. Irani DN. Brain-derived gangliosides induce cell cycle arrest in a murine T cell line. J Neuroimmunol. 1998;87(1-2):11-16.

14. Irani DN, Lin KI, Griffin DE. Brain-derived gangliosides regulate the cytokine production and proliferation of activated T cells. J Immunol. 1996; 157(10):4333-4340.

15. Kwidzinski E, et al. Self-tolerance in the immune privileged CNS: lessons from the entorhinal cortex lesion model. J Neural Transm Suppl. 2003;(65):29-49.

16. Malipiero U, et al. TGFbeta receptor II gene deletion in leucocytes prevents cerebral vasculitis in bacterial meningitis. Brain. 2006;129(pt 9):2404-2415.

17. Strle K, et al. Interleukin-10 in the brain. Crit Rev Immunol. 2001;21(5):427-449.

18. Kawai T, Akira S. The role of pattern-recognition receptors in innate immunity: update on Toll-like receptors. Nat Immunol. 2010;11(5):373-384.

19. Kufer TA, Sansonetti PJ. Sensing of bacteria: NOD a lonely job. Curr Opin Microbiol. 2007;10(1):62-69.

20. Kufer TA, Sansonetti PJ. NLR functions beyond pathogen recognition. Nat Immunol. 2011; 12(2):121-128.

21. O'Neill LA. TLRs: Professor Mechnikov, sit on your hat. Trends Immunol. 2004;25(12):687-693.

22. Martinon F, Tschopp J. Inflammatory caspases: linking an intracellular innate immune system to autoinflammatory diseases. Cell. 2004;117(5):561-574.

23. Chakraborty S, Kaushik DK, Gupta M, Basu A. Inflammasome signaling at the heart of central nervous system pathology. J Neurosci Res. 2010;88(8):1615-1631.

24. Stutz A, Golenbock DT, Latz E. Inflammasomes: too big to miss. J Clin Invest. 2009;119(12):3502-3511.

25. Martinon F, Gaide O, Petrilli V, Mayor A, Tschopp J. NALP inflammasomes: a central role in innate immunity. Semin Immunopathol. 2007;29(3):213-229.

26. Petrilli V, Papin S, Dostert C, Mayor A, Martinon F, Tschopp J. Activation of the NALP3 inflammasome is triggered by low intracellular potassium concentration. Cell Death Differ. 2007;14(9):1583-1589.

27. Oboki K, et al. IL-33 is a crucial amplifier of innate rather than acquired immunity. Proc Natl Acad Sci US A. 2010;107(43):18581-18586

28. Han P, Mi WL, Wang YQ. Research progress on interleukin-33 and its roles in the central nervous system. Neurosci Bull. 2011;27(5):351-357.

29. Palmer G, Gabay C. Interleukin-33 biology with potential insights into human diseases. Nat Rev Rheumatol. 2011;7(6):321-329.

30. Bianchi ME. DAMPs, PAMPs and alarmins: all we need to know about danger. J Leukoc Biol. 2007; 81(1):1-5.

31. Weber AN, et al. Binding of the Drosophila cytokine Spatzle to Toll is direct and establishes signaling. Nat Immunol. 2003;4(8):794-800.

32. Kawai T, Akira S. The roles of TLRs, RLRs and NLRs in pathogen recognition. Int Immunol. 2009; 21(4):317-337.

33. Lehnardt $S$. Innate immunity and neuroinflammation in the CNS: the role of microglia in Tolllike receptor-mediated neuronal injury. Glia. 2010; 58(3):253-263.

34. Downes CE, Crack PJ. Neural injury following stroke: are Toll-like receptors the link between the immune system and the CNS? BrJ Pharmacol. 2010; 160(8):1872-1888.

35. Gorina R, Santalucia T, Petegnief V, Ejarque-Ortiz A, Saura J, Planas AM. Astrocytes are very sensitive to develop innate immune responses to lipid-carried short interfering RNA. Glia. 2009;57(1):93-107.

36. Halle A, et al. The NALP3 inflammasome is involved in the innate immune response to amyloid-beta. Nat Immunol. 2008;9(8):857-865.

37. Salminen A, Ojala J, Suuronen T, Kaarniranta K, Kauppinen A. Amyloid-beta oligomers set fire to inflammasomes and induce Alzheimer's pathology. J Cell Mol Med. 2008;12(6A):2255-2262.

38. Blamire AM, Anthony DC, Rajagopalan B, Sibson NR, Perry VH, Styles P. Interleukin-1beta-induced changes in blood-brain barrier permeability, appar- 
ent diffusion coefficient, and cerebral blood volume in the rat brain: a magnetic resonance study. J Neurosci. 2000;20(21):8153-8159.

39. Zhang Q, et al. Circulating mitochondrial DAMPs cause inflammatory responses to injury. Nature. 2010;464(7285):104-107.

40. Gris D, et al. NLRP3 plays a critical role in the development of experimental autoimmune encephalomyelitis by mediating Th1 and Th17 responses. J Immunol. 2010;185(2):974-981.

41. Dixon SJ, Yu R, Panupinthu N, Wilson JX. Activation of $\mathrm{P} 2$ nucleotide receptors stimulates acid efflux from astrocytes. Glia. 2004;47(4):367-376.

42. Qu Y, Franchi L, Nunez G, Dubyak GR. Nonclassical IL-1 beta secretion stimulated by P2X7 receptors is dependent on inflammasome activation and correlated with exosome release in murine macrophages. J Immunol. 2007;179(3):1913-1925.

43. Ransohoff RM, Cardona AE. The myeloid cells of the central nervous system parenchyma. Nature. 2010;468(7321):253-262.

44. Ginhoux F, et al. Fate mapping analysis reveals that adult microglia derive from primitive macrophages. Science. 2010;330(6005):841-845.

45. Ajami B, Bennett JL, Krieger C, Tetzlaff W, Rossi FM. Local self-renewal can sustain CNS microglia maintenance and function throughout adult life. Nat Neurosci. 2007;10(12):1538-1543.

46. Hanisch UK, Kettenmann H. Microglia: active sensor and versatile effector cells in the normal and pathologic brain. Nat Neurosci. 2007;10(11):1387-1394.

47. Ransohoff RM, Perry VH. Microglial physiology: unique stimuli, specialized responses. Annu Rev Immunol. 2009;27:119-145.

48. del Rio-Hortega P, Penfield W. Cerebral cicatrix: The reaction of neuroglia and microglia to brain wounds. Bull Johns Hopkins Hospital. 1892;41:278-303.

49. Geissmann F, Gordon S, Hume DA, Mowat AM, Randolph GJ. Unravelling mononuclear phagocyte heterogeneity. Nat Rev Immunol. 2010;10(6):453-460.

50. Gordon S, Taylor PR. Monocyte and macrophage heterogeneity. Nat Rev Immunol. 2005;5(12):953-964.

51. Ajami B, Bennett JL, Krieger C, McNagny KM, Rossi FM. Infiltrating monocytes trigger EAE progression, but do not contribute to the resident microglia pool. Nat Neurosci. 2011;14(9):1142-1149.

52. Prinz M, Priller J, Sisodia SS, Ransohoff RM. Heterogeneity of central nervous system myeloid cells and their role in neurodegeneration. Nat Neurosci. 2011;14(10):1227-1235.

53. Palin K, Cunningham C, Forse P, Perry VH, Platt N. Systemic inflammation switches the inflammatory cytokine profile in CNS Wallerian degeneration. Neurobiol Dis. 2008;30(1):19-29.

54. Perry VH, Cunningham C, Holmes C. Systemic infections and inflammation affect chronic neurodegeneration. Nat Rev Immunol. 2007;7(2):161-167.

55. Cunningham C, Campion S, Teeling J, Felton L, Perry VH. The sickness behaviour and CNS inflammatory mediator profile induced by systemic challenge of mice with synthetic double-stranded RNA (poly I:C). Brain Behav Immun. 2007;21(4):490-502.

56. Cunningham C, Wilcockson DC, Campion S, Lunnon $\mathrm{K}$, Perry VH. Central and systemic endotoxin challenges exacerbate the local inflammatory response and increase neuronal death during chronic neurodegeneration. J Neurosci. 2005;25(40):9275-9284.

57. Perry VH. The influence of systemic inflammation on inflammation in the brain: implications for chronic neurodegenerative disease. Brain Behav Immun. 2004;18(5):407-413.

58. Mirrione MM, et al. Microglial ablation and lipopolysaccharide preconditioning affects pilocarpine-induced seizures in mice. Neurobiol Dis. 2010; 39(1):85-97

59. Weinstein JR, Koerner IP, Moller T. Microglia in ischemic brain injury. Future Neurol. 2010;5(2):227-246.

60. Shpargel KB, Jalabi W, Jin Y, Dadabayev A, Penn
MS, Trapp BD. Preconditioning paradigms and pathways in the brain. Cleve Clin J Med. 2008; 75(suppl 2):S77-S82.

61. Rosenzweig HL, Lessov NS, Henshall DC, Minami M, Simon RP, Stenzel-Poore MP. Endotoxin preconditioning prevents cellular inflammatory response during ischemic neuroprotection in mice. Stroke. 2004;35(11):2576-2581.

62 . Boillee $\mathrm{S}$, et al. Onset and progression in inherited ALS determined by motor neurons and microglia. Science. 2006;312(5778):1389-1392.

63. Cardona AE, et al. Control of microglial neurotoxicity by the fractalkine receptor. Nat Neurosci. 2006;9(7):917-924.

64. Harrison JK, et al. Role for neuronally derived fractalkine in mediating interactions between neurons and CX3CR1-expressing microglia. Proc Natl Acad Sci US A. 1998;95(18):10896-10901.

65. Lee $S$, et al. CX3CR1 deficiency alters microglial activation and reduces beta-amyloid deposition in two Alzheimer's disease mouse models. Am J Pathol. 2010;177(5):2549-2562

66. Liu Z, Condello C, Schain A, Harb R, Grutzendler J. CX3CR1 in microglia regulates brain amyloid deposition through selective protofibrillar amyloid-\{beta\} phagocytosis. J Neurosci. 2010;30(50):17091-17101.

67. Bhaskar K, Konerth M, Kokiko-Cochran ON, Cardona A, Ransohoff RM, Lamb BT. Regulation of tau pathology by the microglial fractalkine receptor. Neuron. 2010;68(1):19-31.

68. Fuhrmann M, et al. Microglial Cx3cr1 knockout prevents neuron loss in a mouse model of Alzheimer's disease. Nat Neurosci. 2010;13(4):411-413.

69. Masters SL, O’Neill LA. Disease-associated amyloid and misfolded protein aggregates activate the inflammasome. Trends Mol Med. 2011;17(5):276-282.

70. Nave KA. Myelination and support of axonal integrity by glia. Nature. 2010;468(7321):244-252

71. Eroglu C, Barres BA. Regulation of synaptic connectivity by glia. Nature. 2010;468(7321):223-231.

72. Attwell D, Buchan AM, Charpak S, Lauritzen M, MacVicar BA, Newman EA. Glial and neuronal control of brain blood flow. Nature. 2010;468(7321):232-243.

73. Chouard T, Gray N. Glia. Nature. 2010;468(7321):213.

74. Smith K. Neuroscience: settling the great glia debate. Nature. 2010;468(7321):160-162.

75. Nash B, et al. Functional duality of astrocytes in myelination. J Neurosci. 2011;31(37):13028-13038.

76. Allaman I, Belanger M, Magistretti PJ. Astrocyteneuron metabolic relationships: for better and for worse. Trends Neurosci. 2011;34(2):76-87.

77. McKimmie CS, Graham GJ. Astrocytes modulate the chemokine network in a pathogen-specific manner. Biochem Biophys Res Commun. 2010; 394(4):1006-1011.

78. Lobsiger CS, Cleveland DW. Glial cells as intrinsic components of non-cell-autonomous neurodegenerative disease. Nat Neurosci. 2007;10(11):1355-1360.

79. Vesce S, Rossi D, Brambilla L, Volterra A. Glutamate release from astrocytes in physiological conditions and in neurodegenerative disorders characterized by neuroinflammation. Int Rev Neurobiol. 2007; 82:57-71

80. Bonifati DM, Kishore U. Role of complement in neurodegeneration and neuroinflammation. $\mathrm{Mol}$ Immunol. 2007;44(5):999-1010.

81. Rostasy K, et al. SDF-1alpha is expressed in astrocytes and neurons in the AIDS dementia complex: an in vivo and in vitro study. J Neuropathol Exp Neurol. 2003;62(6):617-626

82. Panenka W, et al. P2X7-like receptor activation in astrocytes increases chemokine monocyte chemoattractant protein-1 expression via mitogen-activated protein kinase. J Neurosci. 2001;21(18):7135-7142.

83. Liu MT, et al. The T cell chemoattractant IFNinducible protein 10 is essential in host defense against viral-induced neurologic disease. J Immunol. 2000;165(5):2327-2330
84. Van Der Voorn P, Tekstra J, Beelen RH, Tensen CP, Van Der Valk P, De Groot CJ. Expression of MCP-1 by reactive astrocytes in demyelinating multiple sclerosis lesions. Am J Pathol. 1999;154(1):45-51.

85 . Conant $\mathrm{K}$, et al. Induction of monocyte chemoattractant protein-1 in HIV-1 Tat-stimulated astrocytes and elevation in AIDS dementia. Proc Natl Acad Sci US A. 1998;95(6):3117-3121.

86. Stoll G, Jander S, Schroeter M. Inflammation and glial responses in ischemic brain lesions. Prog Neurobiol. 1998;56(2):149-171.

87. Majumder S, Zhou LZ, Ransohoff RM. Transcriptional regulation of chemokine gene expression in astrocytes. J Neurosci Res. 1996;45(6):758-769.

88. Glabinski AR, et al. Chemokine monocyte chemoattractant protein- 1 is expressed by astrocytes after mechanical injury to the brain. J Immunol. 1996; 156(11):4363-4368.

89. Ransohoff RM, et al. Astrocyte expression of mRNA encoding cytokines IP-10 and JE/MCP-1 in experimental autoimmune encephalomyelitis. FASEB J. 1993;7(6):592-600.

90. Rossi D, Volterra A. Astrocytic dysfunction: insights on the role in neurodegeneration. Brain Res Bull. 2009;80(4-5):224-232.

91. Bezzi P, et al. CXCR4-activated astrocyte glutamate release via TNFalpha: amplification by microglia triggers neurotoxicity. Nat Neurosci. 2001;4(7):702-710.

92. Saijo K, et al. A Nurr1/CoREST pathway in microglia and astrocytes protects dopaminergic neurons from inflammation-induced death. Cell. 2009; 137(1):47-59.

93. Klotz L, et al. The nuclear receptor PPAR gamma selectively inhibits Th17 differentiation in a T cellintrinsic fashion and suppresses CNS autoimmunity. J Exp Med. 2009;206(10):2079-2089.

94. Brambilla $\mathrm{R}$, et al. Inhibition of astroglial nuclear factor kappaB reduces inflammation and improves functional recovery after spinal cord injury. $J$ Exp Med. 2005;202(1):145-156

95. Dvoriantchikova G, et al. Inactivation of astroglial NF-kappa B promotes survival of retinal neurons following ischemic injury. Eur J Neurosci. 2009; 30(2):175-185.

96. Fu ES, Zhang YP, Sagen J, Yang ZQ, Bethea JR. Transgenic glial nuclear factor-kappa B inhibition decreases formalin pain in mice. Neuroreport. 2007;18(7):713-717

97. Brambilla $\mathrm{R}$, et al. Transgenic inhibition of astroglial NF-kappa B improves functional outcome in experimental autoimmune encephalomyelitis by suppressing chronic central nervous system inflammation. J Immunol. 2009;182(5):2628-2640.

98. Brambilla R, et al. Transgenic inhibition of astroglial NF-kappa B leads to increased axonal sparing and sprouting following spinal cord injury. $\mathrm{J} \mathrm{Neu}$ rochem. 2009;110(2):765-778.

99. Hauwel M, Furon E, Canova C, Griffiths M, Neal J, Gasque P. Innate (inherent) control of brain infection, brain inflammation and brain repair: the role of microglia, astrocytes, "protective" glial stem cells and stromal ependymal cells. Brain Res Brain Res Rev. 2005;48(2):220-233.

100. Tsunoda I, Lane TE, Blackett J, Fujinami RS. Distinct roles for IP-10/CXCL10 in three animal models, Theiler's virus infection, EAE, and MHV infection, for multiple sclerosis: implication of differing roles for IP-10. Mult Scler. 2004;10(1):26-34.

101.Held KS, Chen BP, Kuziel WA, Rollins BJ, Lane TE. Differential roles of CCL2 and CCR2 in host defense to coronavirus infection. Virology. 2004; 329(2):251-260.

102. Trifilo MJ, Bergmann CC, Kuziel WA, Lane TE. CC chemokine ligand 3 (CCL3) regulates CD8(+)-Tcell effector function and migration following viral infection. J Virol. 2003;77(7):4004-4014.

103.Trifilo MJ, Bergmann CC, Kuziel WA, Lane TE. CC chemokine ligand 3 (CCL3) regulates CD8(+)-T- 
cell effector function and migration following viral infection. J Virol. 2003;77(7):4004-4014.

104. Chen BP, Kuziel WA, Lane TE. Lack of CCR2 results in increased mortality and impaired leukocyte activation and trafficking following infection of the central nervous system with a neurotropic coronavirus. J Immunol. 2001;167(8):4585-4592.

105. Hauser SL, Oksenberg JR. The neurobiology of multiple sclerosis: genes, inflammation, and neurodegeneration. Neuron. 2006;52(1):61-76.

106. Mix E, Meyer-Rienecker H, Zettl UK. Animal models of multiple sclerosis for the development and validation of novel therapies - potential and limitations. J Neurol. 2008;255(suppl 6):7-14.

107. Glabinski AR, Tani M, Tuohy VK, Ransohoff RM. Murine experimental autoimmune encephalomyelitis: a model of immune-mediated inflammation and multiple sclerosis. Methods Enzymol. 1997; 288:182-190.

108. Sawcer S, et al. Genetic risk and a primary role for cell-mediated immune mechanisms in multiple sclerosis. Nature. 2011;476(7359):214-219.

109. Severson C, Hafler DA. T-cells in multiple sclerosis. Results Probl Cell Differ. 2010;51:75-98.

110. Martin R, Voskuhl R, Flerlage M, McFarlin DE, McFarland HF. Myelin basic protein-specific T-cell responses in identical twins discordant or concordant for multiple sclerosis. Ann Neurol. 1993; 34(4):524-535.

111.Dornmair K, Goebels N, Weltzien HU, Wekerle H, Hohlfeld R. T-cell-mediated autoimmunity: novel techniques to characterize autoreactive T-cell receptors. Am J Pathol. 2003;163(4):1215-1226.

112. Codarri L, et al. RORgammat drives production of the cytokine GM-CSF in helper T cells, which is essential for the effector phase of autoimmune neuroinflammation. Nat Immunol. 2011;12(6):560-567.

113. Kroenke MA, Chensue SW, Segal BM. EAE mediated by a non-IFN-gamma/non-IL-17 pathway. Eur J Immunol. 2010;40(8):2340-2348.

114.Sonderegger I, Iezzi G, Maier R, Schmitz N, Kurrer M, Kopf M. GM-CSF mediates autoimmunity by enhancing IL-6-dependent Th17 cell development and survival. J Exp Med. 2008;205(10):2281-2294.

115. Li J, et al. Differential expression and regulation of IL-23 and IL-12 subunits and receptors in adult mouse microglia. J Neurol Sci. 2003;215(1-2):95-103.

116. Heppner FL, et al. Experimental autoimmune encephalomyelitis repressed by microglial paralysis. Nat Med. 2005;11(2):146-152.

117. Burmester GR, Feist E, Sleeman MA, Wang B, White B, Magrini F. Mavrilimumab, a human monoclonal antibody targeting GM-CSF receptor-alpha, in subjects with rheumatoid arthritis: a randomised, double-blind, placebo-controlled, phase I, first-in-human study. Ann Rheum Dis. 2011;70(9):1542-1549.

118. Borregaard N. Neutrophils, from marrow to microbes. Immunity. 2010;33(5):657-670.

119. Nathan C. Neutrophils and immunity: challenges and opportunities. Nat Rev Immunol. 2006;6(3):173-182.

120. Mantovani A, Cassatella MA, Costantini C, Jaillon S. Neutrophils in the activation and regulation of innate and adaptive immunity. Nat Rev Immunol. 2011;11(8):519-531.

121.Ishii D, Schenk AD, Baba S, Fairchild RL. Role of TNFalpha in early chemokine production and leukocyte infiltration into heart allografts. Am J Transplant. 2010;10(1):59-68.

122. Halloran PF, Fairchild RL. The puzzling role of CXCR3 and its ligands in organ allograft rejection. Am J Transplant. 2008;8(8):1578-1579.

123. Morita K, et al. Early chemokine cascades in murine cardiac grafts regulate $T$ cell recruitment and progression of acute allograft rejection. J Immunol. 2001;167(5):2979-2984.
124. Infante-Duarte C, Horton HF, Byrne MC, Kamradt T. Microbial lipopeptides induce the production of IL-17 in Th cells. J Immunol. 2000;165(11):6107-6115.

125.Koedel U, Klein M, Pfister HW. New understandings on the pathophysiology of bacterial meningitis. Curr Opin Infect Dis. 2010;23(3):217-223.

126. Openshaw $\mathrm{H}$, et al. Multiple sclerosis flares associated with recombinant granulocyte colony-stimulating factor. Neurology. 2000;54(11):2147-2150.

127. Holman DW, Klein RS, Ransohoff RM. The bloodbrain barrier, chemokines and multiple sclerosis. Biochim Biophys Acta. 2011;1812(2):220-230.

128. Wingerchuk DM, Lennon V, Lucchinetti CF, Pittock SJ, Weinshenker BG. The spectrum of neuromyelitis optica. Lancet Neurol. 2007;6(9):805-815.

129. Maatta JA, Sjoholm UR, Nygardas PT, Salmi AA, Hinkkanen AE. Neutrophils secreting tumor necrosis factor alpha infiltrate the central nervous system of BALB/c mice with experimental autoimmune encephalomyelitis. J Neuroimmunol. 1998; 90(2):162-175.

130.McColl SR, Staykova MA, Wozniak A, Fordham $\mathrm{S}$, Bruce J, Willenborg DO. Treatment with antigranulocyte antibodies inhibits the effector phase of experimental autoimmune encephalomyelitis. J Immunol. 1998;161(11):6421-6426.

131. Carlson T, Kroenke M, Rao P, Lane TE, Segal B. The Th17-ELR+ CXC chemokine pathway is essential for the development of central nervous system autoimmune disease. J Exp Med. 2008;205(4):811-823.

132. Liu L, Darnall L, Hu T, Choi K, Lane TE, Ransohoff RM. Myelin repair is accelerated by inactivating CXCR2 on nonhematopoietic cells. J Neurosci. 2010;30(27):9074-9083.

133. Sayed BA, Christy AL, Walker ME, Brown MA. Meningeal mast cells affect early $T$ cell central nervous system infiltration and blood-brain barrier integrity through TNF: a role for neutrophil recruitment? Jimmunol. 2010;184(12):6891-6900.

134.Magliozzi R, et al. Meningeal B-cell follicles in secondary progressive multiple sclerosis associate with early onset of disease and severe cortical pathology. Brain. 2007;130(pt 4):1089-1104.

135.Serafini B, Rosicarelli B, Magliozzi R, Stigliano E, Aloisi F. Detection of ectopic B-cell follicles with germinal centers in the meninges of patients with secondary progressive multiple sclerosis. Brain Pathol. 2004;14(2):164-174.

136. Galli SJ, Tsai M. Mast cells in allergy and infection: versatile effector and regulatory cells in innate and adaptive immunity. Eur J Immunol. 2010; 40(7):1843-1851.

137. Rao KN, Brown MA. Mast cells: multifaceted immune cells with diverse roles in health and disease. Ann N Y Acad Sci. 2008;1143:83-104

138. Malaviya R, Ikeda T, Ross E, Abraham SN. Mast cell modulation of neutrophil influx and bacterial clearance at sites of infection through TNF-alpha. Nature. 1996;381(6577):77-80.

139.Schramm R, Thorlacius H. Neutrophil recruitment in mast cell-dependent inflammation: inhibitory mechanisms of glucocorticoids. Inflamm Res. 2004;53(12):644-652.

140.Nakamura Y, et al. Mast cells mediate neutrophil recruitment and vascular leakage through the NLRP3 inflammasome in histamine-independent urticaria. J Exp Med. 2009;206(5):1037-1046.

141.Ibrahim MZ, Reder AT, Lawand R, Takash W, Sallouh-Khatib S. The mast cells of the multiple sclerosis brain. J Neuroimmunol. 1996;70(2):131-138.

142.Neumann J. Ueber das Vorkommen der sogneannten "Mastzellen" bei pathologischen Veraenderungen des Gehirns. Arch Pathol Anat Physiol Virchows. 1890;122:378-381.

143.Lock C, et al. Gene-microarray analysis of mul- tiple sclerosis lesions yields new targets validated in autoimmune encephalomyelitis. Nat Med. 2002; 8(5):500-508

144. Rozniecki JJ, Hauser SL, Stein M, Lincoln R, Theoharides TC. Elevated mast cell tryptase in cerebrospinal fluid of multiple sclerosis patients. Ann Neurol. 1995;37(1):63-66.

145. Tuomisto L, Kilpelainen H, Riekkinen P. Histamine and histamine-N-methyltransferase in the CSF of patients with multiple sclerosis. Agents Actions. 1983;13(2-3):255-257.

146.Lindsberg PJ, Strbian D, Karjalainen-Lindsberg ML. Mast cells as early responders in the regulation of acute blood-brain barrier changes after cerebral ischemia and hemorrhage. J Cereb Blood Flow Metab. 2010;30(4):689-702.

147. Tanzola MB, Robbie-Ryan M, Gutekunst CA, Brown MA. Mast cells exert effects outside the central nervous system to influence experimental allergic encephalomyelitis disease course. J Immunol. 2003;171(8):4385-4391.

148. Nakano T, et al. Pillars article: fate of bone marrow-derived cultured mast cells after intracutaneous, intraperitoneal, and intravenous transfer into genetically mast cell-deficient w/wv mice. Evidence that cultured mast cells can give rise to both connective tissue type and mucosal mast cells. J Immunol. 2009;183(11):6863-6881.

149. Grimbaldeston MA, Chen CC, Piliponsky AM, Tsai M, Tam SY, Galli SJ. Mast cell-deficient W-sash c-kit mutant Kit W-sh/W-sh mice as a model for investigating mast cell biology in vivo. Am J Pathol. 2005;167(3):835-848

150.Bennett JL, et al. Bone marrow-derived mast cells accumulate in the central nervous system during inflammation but are dispensable for experimental autoimmune encephalomyelitis pathogenesis. J Immunol. 2009;182(9):5507-5514.

151.Li H, et al. Kit (W-sh) mice develop earlier and more severe experimental autoimmune encephalomyelitis due to absence of immune suppression. J Immunol. 2011;187(1):274-282.

152.Piconese S, et al. Exacerbated experimental autoimmune encephalomyelitis in mast-cell-deficient Kit W-sh/W-sh mice. Lab Invest. 2011;91(4):627-641.

153.Secor VH, Secor WE, Gutekunst CA, Brown MA. Mast cells are essential for early onset and severe disease in a murine model of multiple sclerosis. J Exp Med. 2000;191(5):813-822.

154.Sayed BA, Walker ME, Brown MA. Cutting edge: mast cells regulate disease severity in a relapsingremitting model of multiple sclerosis. J Immunol. 2011;186(6):3294-3298.

155. Bartholomaus I, et al. Effector T cell interactions with meningeal vascular structures in nascent autoimmune CNS lesions. Nature. 2009;462(7269):94-98.

156. Kirmi O, Sheerin F, Patel N. Imaging of the meninges and the extra-axial spaces. Semin Ultrasound CT MR. 2009;30(6):565-593.

157. Patel N, Kirmi O. Anatomy and imaging of the normal meninges. Semin Ultrasound CT MR. 2009; 30(6):559-564.

158. Kivisakk P, et al. Localizing central nervous system immune surveillance: meningeal antigen-presenting cells activate $T$ cells during experimental autoimmune encephalomyelitis. Ann Neurol. 2009;65(4):457-469.

159.Abraham SN, St. John AL. Mast cell-orchestrated immunity to pathogens. Nat Rev Immunol. 2010; 10(6):440-452.

160.Dudeck A, et al. Mast cells are key promoters of contact allergy that mediate the adjuvant effects of haptens. Immunity. 2011;34(6):973-984.

161.Kettenmann H, Hanisch UK, Noda M, Verkhratsky A. Physiology of microglia. Physiol Rev. 2011; 91(2):461-553. 